\title{
Urgent Chemotherapy Successfully Rescues a Near Death Patient of Acute Intracranial Hypertension Caused by Intracranial Myeloid Sarcoma
}

This article was published in the following Dove Press journal: OncoTargets and Therapy

\author{
Li Zhou (iD) ${ }^{1, *}$ \\ Xinhui Zhang ${ }^{2, *}$ \\ Shanglong Feng ${ }^{2}$ \\ $\mathrm{Na}$ Zhao' \\ Xing $\mathrm{Hu}^{\prime}$ \\ Liangliang Huang ${ }^{3}$ \\ Changcheng Zheng $\mathbb{D}^{1,2}$ \\ 'Department of Hematology, The First \\ Affiliated Hospital of USTC, Division of \\ Life Sciences and Medicine, University of \\ Science and Technology of China, Hefei, \\ People's Republic of China; ${ }^{2}$ Department \\ of Hematology, Anhui Provincial Hospital, \\ Anhui Medical University, Hefei, People's \\ Republic of China; ${ }^{3}$ Clinic Pathology \\ Center, The First Affiliated Hospital of \\ USTC, Division of Life Sciences and \\ Medicine, University of Science and \\ Technology of China, Hefei, People's \\ Republic of China
}

*These authors contributed equally to this work
Correspondence: Changcheng Zheng Department of Hematology, The First Affiliated Hospital of USTC, Division of Life Sciences and Medicine, University of Science and Technology of China, Lujiang Road No. 17, Hefei 23000I, People's

Republic of China

$\mathrm{Tel} / \mathrm{Fax}+86-55 \mathrm{I}-62284476$

Email zhengchch II23@ustc.edu.cn

\begin{abstract}
Intracranial myeloid sarcoma is a very rare disease with poor prognosis. We report a case of a 28 -year-old male patient who was admitted with intense headache, vision disturbance and severe vomiting in June 2017. He had a history of neurosurgical tumor resection operation in April 2017, and the pathological diagnosis was intracranial myeloid sarcoma. Bone marrow aspirate and biopsy had been conducted in May 2017, which demonstrated 5.5\% blasts expressing CD13, CD33, CD34, CD117 and MPO, and the cytogenetic analysis demonstrated $\mathrm{t}(8 ; 21)(\mathrm{q} 22 ; \mathrm{q} 22)$, and molecular studies showed a positive RUNX1-RUNX1T1 rearrangement. The diagnosis of acute myeloid leukemia (AML) with $\mathrm{t}(8 ; 21)$ (q22; q22)/RUNX1-RUNX1T1 was made, however, the patient refused to receive any systemic chemotherapy. Emergency cranial $\mathrm{CT}$ demonstrated a circular hyperdense mass $(54 \mathrm{~mm} \times 37 \mathrm{~mm})$, which was surrounded by hypodense peritumoral edema in the left cerebellar hemisphere, and the density of the lesions was uniform and the margin was clear. Idarubicin $\left(12 \mathrm{mg} / \mathrm{m}^{2} \cdot \mathrm{d} \times 3\right.$ days $)$ combined with high-dose cytarabine $\left(2 \mathrm{~g} / \mathrm{m}^{2} \mathrm{q} 12 \mathrm{~h} \times 3\right.$ days $)$ was initiated for emergency chemotherapy. All of the above symptoms disappeared at the end of chemotherapy. On the first day after chemotherapy, the cranial CT indicated that the cranial lesion was markedly reduced $(20 \mathrm{~mm} \times 15 \mathrm{~mm})$, and on the sixth day after chemotherapy, the lesion was completely disappeared. Currently, there are no clear guidelines for the treatment of intracranial myeloid sarcoma, and our treatment approaches could provide a reference for this disease with such emergency situation.
\end{abstract}

Keywords: myeloid sarcoma, acute myeloid leukemia, chemotherapy

\section{Introduction}

Intracranial myeloid sarcoma is a very rare disease presenting with or without bone marrow involvement ${ }^{1}$. The optimal treatment remains unknown. Conventional acute myeloid leukemia (AML) -like chemotherapy, radiotherapy, and surgery are the currently recommended strategies in controlling the disease progress ${ }^{2}$. However, the optimal treatment for intracranial myeloid sarcoma remains unknown. In this paper we report a case of successful rescue of intracranial myeloid sarcoma with acute intracranial hypertension through intensified induction chemotherapy of idarubicin $\left(12 \mathrm{mg} / \mathrm{m}^{2} \cdot \mathrm{d} \times 3\right.$ days $)$ combined with high-dose cytarabine $\left(2 \mathrm{~g} / \mathrm{m}^{2} \mathrm{q} 12 \mathrm{~h} \times 3\right.$ days), and we also did a literature review of the published data of intracranial myeloid sarcoma, which mainly focuses on how to choose the optimal treatment for such patients. 


\section{Case Presentation}

A 28-year-old male patient was admitted to the hematological department at our hospital in June 2017, with a 4-day history of intense headache, worsening blurred vision and severe vomiting. The patient had a history of neurosurgical operation with resection of tumor involving the regions of torcular herophili and left transverse sinus in April 2017. The pathological findings of the tumor specimen led to a diagnosis of intracranial myeloid sarcoma. Preoperative magnetic resonance imaging (MRI) (Figure 1) and Postoperative cranial computed tomography (CT) indicated complete resection of the lesion (Supplement Figures 1 and 2). A diagnostic lumbar puncture showed no malignant cells, and methotrexate $10 \mathrm{mg}$, cytarabine $50 \mathrm{mg}$ and dexamethasone $5 \mathrm{mg}$ were given intrathecally. Bone marrow aspirate and biopsy had been conducted in May 2017, which demonstrated 5.5\% blasts expressing CD13, CD33, CD34, CD117 and MPO, and the cytogenetic analysis demonstrated $\mathrm{t}(8 ; 21)(\mathrm{q} 22 ; \mathrm{q} 22)$, and molecular studies showed a positive RUNX1RUNX1T1 rearrangement, but negative for c-KIT, FLT3, NPM1, or CEBPA mutations. The diagnosis of AML with $\mathrm{t}(8 ; 21)$ (q22; q22)/RUNX1-RUNX1T1 was made. However, the patient refused to receive any systemic chemotherapy.

Headache appeared on 60-day after the surgical operation, and aggravated gradually, accompanied by blurred vision and severe vomiting. Blood routine was normal at admission. After admission, the emergency cranial CT indicated a circular hyperdense mass $(54 \mathrm{~mm} \times 37 \mathrm{~mm})$, which was surrounded by hypodense peritumoral edema in the left cerebellar hemisphere,

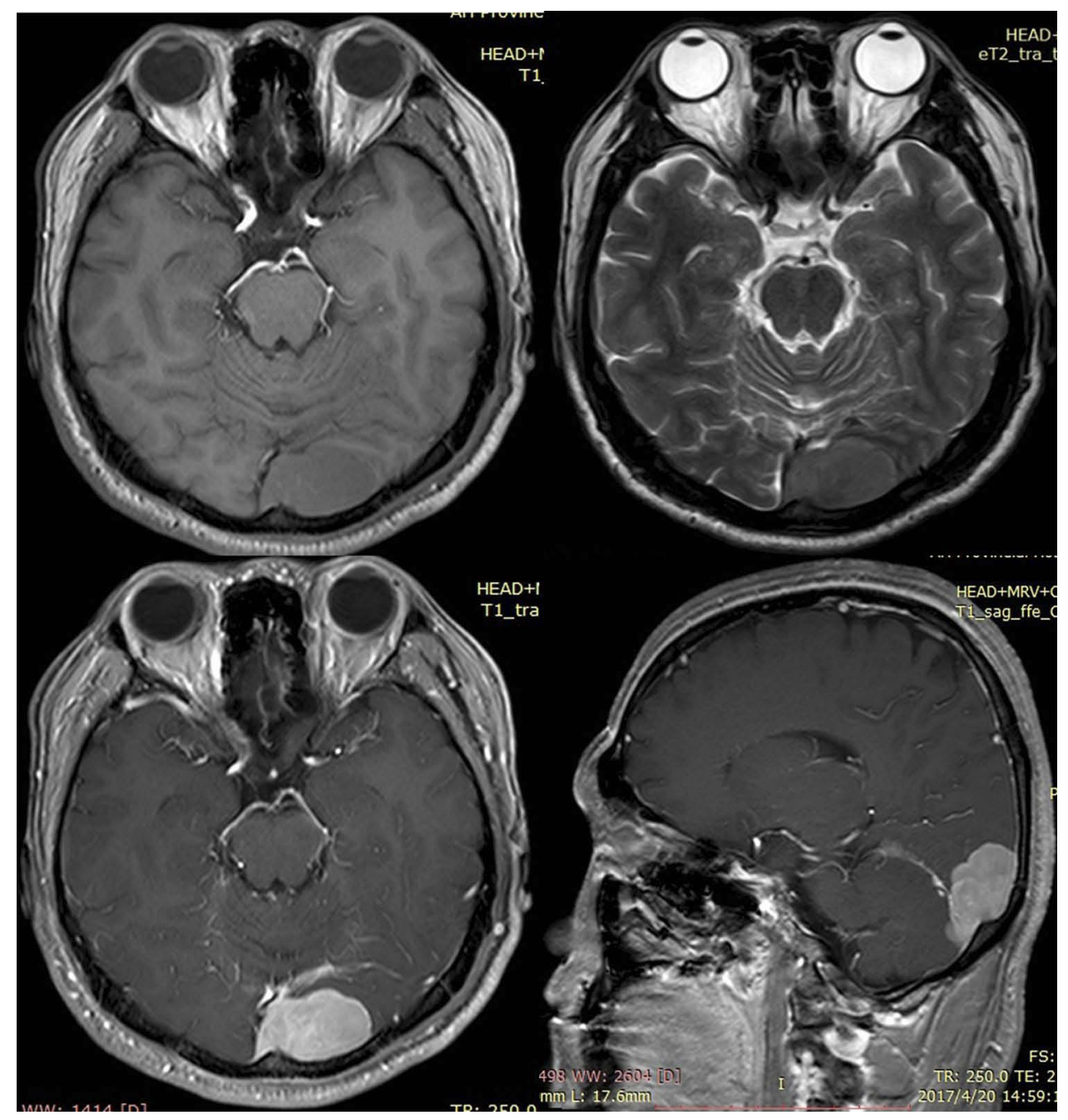

Figure I The MRI of incipient intracranial myeloid sarcoma of the patient before operation. In the left occipital region, there was a kind of circular broad base with slightly longer TI and short T2 signal shadow, with clear boundary and a size of about $23 \times 37 \mathrm{~mm}$. The internal signal was uniform, which was enhanced uniformly. 


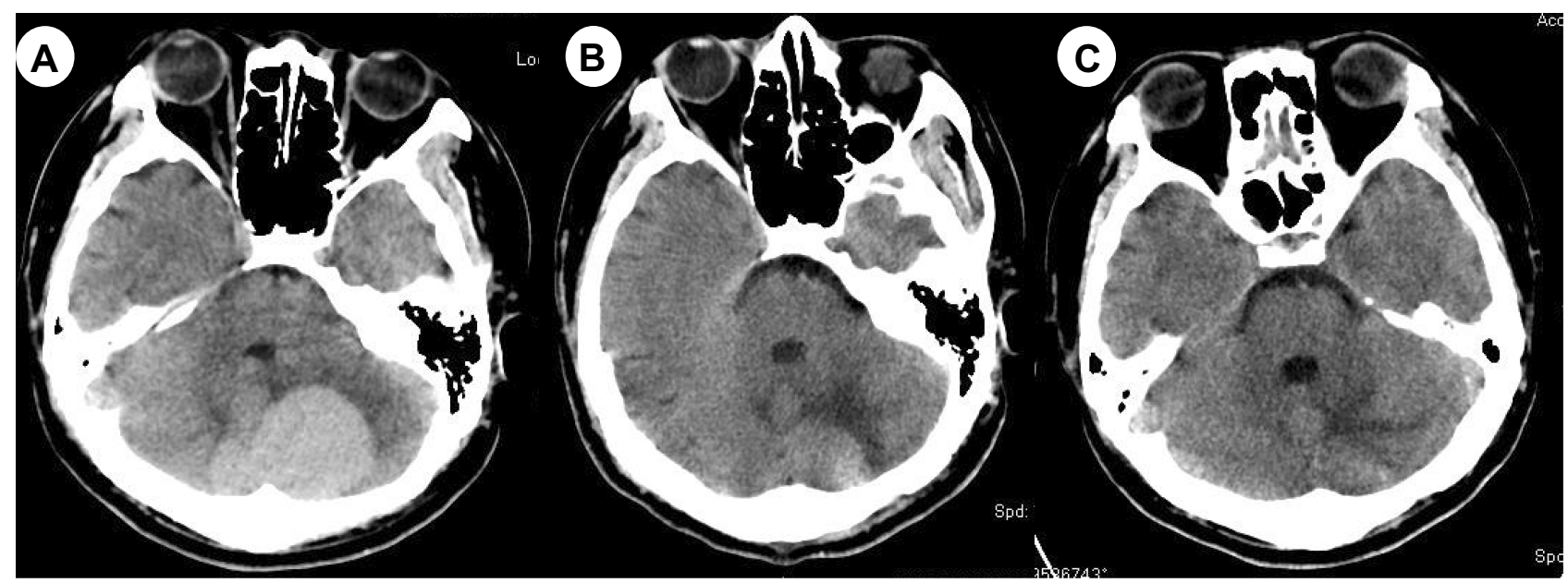

Figure 2 Recurrent intracranial myeloid sarcoma before and after chemotherapy. The emergency cranial CT demonstrated a circular hyperdense mass $(54 \mathrm{~mm} \times 37 \mathrm{~mm})$ in the left cerebellar hemisphere at the admission $(\mathbf{A})$. Cranial CT indicated that the mass was markedly reduced $(20 \mathrm{~mm} \times 15 \mathrm{~mm})$ on the first day after chemotherapy $(\mathbf{B})$, and the mass was completely disappeared on the sixth day after chemotherapy (C).

and the density of the lesions was uniform and the margin was clear (Figure 2A).

The recurrence of intracranial myeloid sarcoma was considered. The patient could die at any time due to acute intracranial hypertension. He refused decompressive craniectomy and emergency cranial radiotherapy to alleviate the symptoms. Idarubicin $\left(12 \mathrm{mg} / \mathrm{m}^{2} . \mathrm{d} \times 3\right.$ days $)$ combined with high-dose cytarabine $\left(2 \mathrm{~g} / \mathrm{m}^{2} . \mathrm{q} 12 \mathrm{~h} \times 3\right.$ days $)$ was initiated for emergency chemotherapy. Mannitol and valproate were used to reduce intracranial pressure and prevent seizures.

Chemotherapy was very effective. On the second day of chemotherapy, the symptoms of headache were obviously relieved, the number of vomiting was reduced, and the vision gradually became clear. All of the above symptoms disappeared at the end of chemotherapy. On the first day after chemotherapy, the cranial CT indicated that the cranial lesion was markedly reduced $(20 \mathrm{~mm} \times 15 \mathrm{~mm})$ (Figure 2B), and on the sixth day after chemotherapy, the lesion was completely disappeared (Figure 2C). The patient experienced 12 days of severe neutropenia. With the support of granulocyte colony stimulating factor (G-CSF), the patient recovered with normal neutrophil on the fourteenth day after chemotherapy. Then the patient received 2 courses of high-dose cytarabine $\left(3 \mathrm{~g} / \mathrm{m}^{2} . \mathrm{q} 12 \mathrm{~h} \times 3\right.$ days) with normal cranial MRI and without any CNS symptoms. Bone marrow aspiration showed morphology complete remission with normal cytogenetics and negative RUNX1-RUNX1T1 arrangement. However, the patient had a very poor compliance and refused any form of medical service after that. And now, the patient has been lost to follow-up.

\section{Discussion and Literature Review}

Intracranial myeloid sarcomas are rare and account for about $3 \%$ of all extramedullary sarcomas. Myeloid sarcoma can be caused by $\mathrm{AML},{ }^{3}$ chronic myeloid leukemia $(\mathrm{CML}),{ }^{4,5}$ chronic myelomonocytic leukemia (CMML), ${ }^{6}$ and primary myelofibrosis (PMF). ${ }^{7-9}$ It has been reported that intracranial myeloid sarcoma may originate from the superficial arachnoid vein wall and extend into the cerebrospinal fluid. The expanded leukemic mass infiltrates into the brain parenchyma through the cortical glial membrane and eventually forms intracerebral tumor. The most common clinical manifestations of intracranial myeloid sarcoma are headache, accompanied by numbness of limbs, blurred vision, epilepsy, etc. Common intracranial myeloid sarcoma gene mutation is $\mathrm{t}(8 ; 21),{ }^{10}$ which is the same as our case. Some others are $t(15 ; 17)$ as in literature. ${ }^{11}$ The misdiagnosis rate of myeloid sarcoma is relatively high. Imaging methods such as CT and MRI can improve the diagnosis and facilitate early treatment, but the definite diagnosis was dependent on the pathology and immunohistochemistry of mass biopsy or surgical specimen. It has been reported that MRI can effectively evaluate the lesion status and treatment effect.

The overall prognosis for extramedullary myeloid sarcoma is poor, with an average survival time of 2.5 to 22 months. Currently, there is no clear or uniform international guideline for this kind of disease. According to the existing literature, most cases are treated with a combination of surgical resection, chemotherapy and/or radiotherapy. Surgical resection and radiotherapy alone do not prevent 
disease recurrence and progression, therefore, do not prolonged the long-term survival. ${ }^{12}$ It has been reported in the literature that intensive chemotherapy regimens, which include anthracyclines and high-dose cytarabine, can certainly improve the survival rate of patients. Hematopoietic stem cell transplantation may be an effective method to improve the long-term survival rate of myeloid sarcoma, especially when patients reach complete remission after intensive chemotherapy. ${ }^{13}$ In our case, chemotherapy achieved the same effect as surgical excision. Similar treatment regimens for subsequent cases are listed in supplement table 1. From which it can be seen that all cases were treated with chemotherapy-based approaches for myeloid sarcoma, and the prognosis of the patients was relatively good. The most common causes of death for myeloid sarcomas are infection, postoperative bleeding, and hernia of the brain due to the short-term enlargement of the tumor. Chemotherapy is as effective as surgical excision, especially with High-dose cytarabine that can penetrate the bloodbrain barrier.

In this reported case, the patient had a recurrence of AML related intracranial myeloid sarcoma. Severe intracranial hypertension was present at the time of admission, and cerebral hernia might occur at any time and lead to death. The patient refused decompressive craniectomy and emergency cranial radiotherapy to alleviate the symptoms. So, chemotherapy, as the current preferred treatment for AML induction, idarubicin $\left(12 \mathrm{mg} / \mathrm{m}^{2} . \mathrm{d} \times 3\right.$ days $)$ combined with high-dose cytarabine $\left(2 \mathrm{~g} / \mathrm{m}^{2} \mathrm{q} 12 \mathrm{~h} \times 3\right.$ days $)$ was initiated for emergency chemotherapy. The lipophilic idarubicin and high-dose cytarabine both can quickly pass through the blood-brain barrier, and penetrate into tumor cells and dissolve tumor cells, thus effectively control the progression of the disease. The use of this combination did not result in a long-term myelosuppression, potentially life-threatening infections and bleeding, and excessive hospital costs or prolonged hospital stays.

\section{Conclusion}

Intracranial myeloid sarcoma is a rare disease with poor prognosis. Currently, there are no clear guidelines for the treatment of relevant diseases. The standard dose of idarubicin $\left(12 \mathrm{mg} / \mathrm{m}^{2} \cdot \mathrm{d} \times 3\right.$ days $)$ and high-dose cytarabine (2$3 \mathrm{~g} / \mathrm{m}^{2}$.q $12 \mathrm{~h} \times 3$ days) both can pass through the blood-brain barrier, thus effectively controlling the progression of the disease without severe complications. Our patient's treatment approach could provide a reference for this disease with such emergency situation.

\section{Acknowledgments}

We would like to thank Dr Wei Wei (Department of Radiology, The First Affiliated Hospital of USTC) for her help taking the CT image pictures. This case has been presented as an abstract for the 24th European Hematology Association (EHA) congress.

\section{Author Contributions}

All authors contributed to data analysis, drafting or revising the article, gave final approval of the version to be published, and agree to be accountable for all aspects of the work.

\section{Ethics Approval}

The Institutional Ethics committee of the First Affiliated Hospital of USTC approved the study, and a written informed consent has been provided by the patient to have the case details and any accompanying images published, in accordance with the Declaration of Helsinki.

\section{Funding}

This work was partly supported by the Fundamental Research Funds for the Central Universities of China (No. WK9110000003), and the Key Projects of Research and Development Program of Anhui Province (201904a07020094).

\section{Disclosure}

The authors declare that there is no conflicts of interest in this work.

\section{References}

1. Campidelli C, Agostinelli C, Stitson R, Pileri SA. Myeloid sarcoma: extramedullary manifestation of myeloid disorders. Am J Clin Pathol. 2009;132(3):426-437. doi:10.1309/AJCP1ZA7HYZKAZHS

2. Bakst RL, Tallman MS, Douer D, Yahalom J. How I treat extramedullary acute myeloid leukemia. Blood. 2011;118(14):3785-3793. doi:10.1182/blood-2011-04-347229

3. Ullman DI, Dorn D, Jones JA, et al. Clinicopathological and molecular characteristics of extramedullary acute myeloid leukaemia. Histopathology. 2019;75(2):185-192. doi:10.1111/his.13864

4. Palejwala AH, O'Connor KP, Shi H, Villeneuve L, Scordino T, Glenn CA. Chronic myeloid leukemia manifested as myeloid sarcoma: review of literature and case report. $J$ Clin Neurosci. 2019;64:269-276. doi:10.1016/j.jocn.2019.04.011

5. Kabadi K, Anoop P, Sandhya V, Murthy GK. Intracranial granulocytic sarcoma as the first presentation of chronic myeloid leukemia in chronic phase. J Neuro Oncol. 2017;134(2):473-474. doi:10.1007/ s11060-017-2541-6

6. Matanes F, AbdelAzeem BMA, Shah G, Reddy V, Saad A, Papadantonakis N. Chronic myelomonocytic leukemia associated with myeloid sarcomas and NPM1 mutation: a case report and literature review. Ther Adv Hematol. 2019;10:2040620719854596. doi: $10.1177 / 2040620719854596$ 
7. Nunes LFM, Rocha AL, Magalhães GHR, et al. Intraoral granulocytic sarcoma as a manifestation of myelofibrosis: a case report and review of the literature. Spec Care Dentist. 2018;38(6):409-420. doi:10.1111/scd.12325

8. Orofino N, Cattaneo D, Bucelli C, et al. An unusual type of myeloid sarcoma localization following myelofibrosis: a case report and literature review. Leuk Res Rep. 2017;8:7-10. doi:10.1016/j.1rr.2017.07.002

9. Kremyanskaya M, Mascarenhas J, Rampal R, Hoffman R. Development of extramedullary sites of leukaemia during ruxolitinib therapy for myelofibrosis. Br J Haematol. 2014;167(1):144-146. doi:10.1111/bjh.12948

10. Pramanik R, Tyagi A, Chopra A, Kumar A, Vishnubhatla S, Bakhshi S. Myeloid sarcoma predicts superior outcome in pediatric AML; can cytogenetics solve the puzzle? Clin Lymphoma Myeloma Leuk. 2018;18(6):e249-e254. doi:10.1016/j.clml.2018.03.013
11. Yamashita T, Nishijima A, Noguchi Y, Narukawa K, Oshikawa G, Takano H. Acute promyelocytic leukemia presenting as recurrent spinal myeloid sarcomas 3 years before developing leukemia: a case report with review of literature. Clin Case Rep. 2019;7 (2):316-321. doi:10.1002/ccr3.1991

12. Antic D, Elezovic I, Milic N, et al. Is there a "gold" standard treatment for patients with isolated myeloid sarcoma? Biomed Pharmacother. 2013;67(1):72-77. doi:10.1016/j.biopha.2012.10. 014

13. Lazzarotto D, Candoni A, Filì C, et al. Clinical outcome of myeloid sarcoma in adult patients and effect of allogeneic stem cell transplantation. Results from a Multicenter Survey. Leuk Res. 2017;53:74-81. doi:10.1016/j.leukres.2016.12.003

\section{Publish your work in this journal}

OncoTargets and Therapy is an international, peer-reviewed, open access journal focusing on the pathological basis of all cancers, potential targets for therapy and treatment protocols employed to improve the management of cancer patients. The journal also focuses on the impact of management programs and new therapeutic agents and protocols on patient perspectives such as quality of life, adherence and satisfaction. The manuscript management system is completely online and includes a very quick and fair peer-review system, which is all easy to use. Visit http://www.dovepress.com/ testimonials.php to read real quotes from published authors. 